\title{
SUBTLETIES IN THE AVERAGING OF HYBRID SYSTEMS WITH APPLICATIONS TO POWER ELECTRONICS
}

\author{
Luigi Iannelli ${ }^{*}$ Karl Henrik Johansson ${ }^{* *}$ Ulf T. Jönsson ${ }^{* * *}$ \\ Francesco Vasca* \\ * Dept. of Engineering, University of Sannio, Benevento, Italy \\ ** Electrical Engineering School, Royal Institute of Technology, \\ Stockholm, Sweden \\ ${ }^{* * *}$ Division of Optimization and Systems Theory, Royal Institute \\ of Technology, Stockholm, Sweden
}

\begin{abstract}
Dither signals are commonly used in electronics for implementing different type of modulations in power converters, which represent a very interesting class of hybrid systems. It was recently shown that a nonsmooth dithered system can be approximated by an averaged system provided that the dither frequency is sufficiently high and that the amplitude distribution function of the dither is absolutely continuous and has bounded derivative. This result is exploited in this paper for power converters. Averaged models corresponding to various shapes of dither signal are analyzed, showing that dither with Lipschitz continuous amplitude distribution function can be used to adapt the equivalent gain of the power converter.
\end{abstract}

\section{INTRODUCTION}

In power electronics a large class of systems can be modeled as hybrid dynamical systems due to the presence of switching devices (for instance diodes or transistors) that "instantaneously" change the dynamical behavior of the system. This class of systems can be adequately represented by hybrid systems or nonlinear differential equations with discontinuous nonlinearities. One of the standard approaches for designing control algorithms for power converters, is to use modulation schemes (e.g. pulse width modulation, PWM). In this case the feedback controlled power converter is a hybrid system with one (possible more) external forcing signal(s).

\footnotetext{
* The work by L. Iannelli and F. Vasca was supported by the European Commission within the SICONOS project IST2001-37172. The work by K. H. Johansson and U. Jönsson was supported by the European Commission within the Network of Excellence HYCON and by the Swedish Research Council. K. H. Johansson was also supported by the Swedish Foundation for Strategic Research.
}

Analysis of forced nonlinear feedback systems can be very difficult. One approach for the analysis is to accept some approximation and base the analysis on the averaged dynamics. Averaging theory provides theoretical justification for this idea in many important cases. Averaging is applied, for example, in studying effects of dither signals that are commonly used to compensate for nonlinearities in feedback control systems. The idea is that by injecting a suitably chosen high-frequency signal in the control loop, the nonlinear sector is effectively narrowed and the system can thereby be stabilized. For the case when the original nonlinearity is Lipschitz continuous, it was shown in the 70s that if the dither frequency is sufficiently high, the behavior of the dithered system will be qualitatively the same as an averaged system, see Zames and Shneydor (1976). However, the Lipschitz continuity assumption on the nonlinearity of the dithered system is often violated in practice. This is the case in many pulse width modulated systems and power converters. These systems can be modeled as nonlinear dithered systems in which the high frequency signal is added 
at the input of a discontinuous nonlinearity of signum or step function type. It is well known that complex phenomena such as bifurcations and chaos can appear in these systems, e.g., di Bernardo and Tse (2002), Tse et al. (2000), Banerjee and Verghese (2001).

Rigorous analysis of nonsmooth systems with dither excitation is complicated. It is only recently that rigorous results on stability and approximation have been obtained, e.g., Gelig and Churilov (1998), Teel et al. (2004), Iannelli et al. (2003), Iannelli et al. (2006). In this paper we will recap and apply to power electronics systems the averaging result in Iannelli et al. (2006). The result shows that a general class of nonsmooth dithered systems can be approximated by a corresponding class of averaged systems, provided that the dither has sufficiently high frequency and its amplitude distribution function is absolutely continuous with bounded derivative. We will discuss how usual modulation schemes (that use dither signals satisfying the averaging result) work well while other modulation schemes that use dither signals not satisfying the assumptions in Iannelli et al. (2006) may fail. Simulations on a buck converter show the effects of different (dither) signals used in the modulation, while experiments show that the averaging result does not hold when zero-slope signals (like square or trapezoidal waveforms) are used for feedback control of a DC motor. The experiments illustrate how it is possible to control the motor shaft at a desired angular position by applying high frequency dither signals that satisfy the assumption of the averaging theorem, while other dither signals that do not fulfill the conditions do not give a stable closed-loop system, despite their high frequency content.

\section{PRELIMINARIES}

Consider the dithered feedback system

$\dot{x}(t)=f_{0}(x(t), t)+\sum_{i=1}^{m} f_{i}(x(t), t) n_{i}\left(g_{i}(x(t), t)-\delta_{i}(t)\right)$,

where $x(0)=x_{0}, f_{i}: \mathbb{R}^{q} \times \mathbb{R} \rightarrow \mathbb{R}^{q}, i=1, \ldots, m$, are assumed to be globally Lipschitz with respect to both $x$ and $t$, and $f_{0}$ is piecewise continuous with respect to $t$, Lipschitz in $x$, and $f_{0}(0, t)=0$ for all $t \geq 0$. Similarly, the functions $g_{i}: \mathbb{R}^{q} \times \mathbb{R} \rightarrow \mathbb{R}, i=1, \ldots, m$, are globally Lipschitz with respect to both $x$ and $t$. The nonlinearities $n_{i}: \mathbb{R} \rightarrow \mathbb{R}, i=1, \ldots, m$, are supposed to be of bounded variation. Each dither signal $\delta_{i}$ : $[0, \infty) \rightarrow \mathbb{R}$ is a $p$-periodic measurable and bounded function.

The averaged system is defined as

$$
\dot{w}(t)=f_{0}(w(t), t)+\sum_{i=1}^{m} f_{i}(w(t), t) N_{i}\left(g_{i}(w(t), t)\right),
$$

where $w(0)=w_{0}, N_{i}$ is the averaged nonlinearity

$$
N_{i}(z) \triangleq \int_{\mathbb{R}} n_{i}(z-\xi) \mathrm{d} F_{\delta_{i}}(\xi)
$$

Here the integral is a Lebesgue-Stieltjes integral and

$$
F_{\delta}(\xi) \triangleq \frac{1}{p} \mu(\{t \in[0, p): \delta(t) \leq \xi\})
$$

is the amplitude distribution function with Lebesgue measure $\mu$ and dither period $p$.

The following averaging result was proved in Iannelli et al. (2006).

Theorem 2.1. (Iannelli et al. (2006)). Consider the dithered system (1) and the averaged system (2) under the following assumptions:

(i) the dithered system has an absolutely continuous solution,

(ii) $f_{i}$ and $g_{i}$ are globally Lipschitz,

(iii) $f_{0}$ is globally Lipschitz with respect to $x$ and $f_{0}(0, t)=0$

(iv) $n_{i}$ is a function of bounded variation,

(v) each dither $\delta_{i}$ is $p$-periodic, $\left|\delta_{i}\right| \leq M_{\delta}$, and has absolutely continuous amplitude distribution function $F_{\delta_{i}}$ with bounded derivative.

Then, the averaged nonlinearities $N_{i}$ are globally Lipschitz continuous and the averaged system (2) has a unique absolutely continuous solution on $[0, \infty)$. Moreover, for any compact set $\mathcal{K} \subset \mathbb{R}^{n}$ and any $T>0$, there exists a positive constant $c(\mathcal{K}, T)$ such that

$\left|x\left(t, x_{0}\right)-w\left(t, x_{0}\right)\right| \leq c(\mathcal{K}, T) p, \quad \forall x_{0} \in \mathcal{K}, t \in[0, T]$.

The main result of this paper is to show that if the conditions of the theorem are violated, then the averaged system does not necessarily approximate the dithered system. It suffices to consider a subclass of the general nonsmooth systems introduced above, that will be discussed in the following and that represent a wide class of power electronics systems.

\section{AVERAGED MODELS OF POWER ELECTRONICS SYSTEMS}

\subsection{PWM power converters}

A typical approach for modeling converters in the power electronics framework is based on the assumption that switches (such as diodes, thyristors, transistors, mosfets) are ideal in the sense that their currentvoltage relationship can be modeled as a piecewise linear characteristic. Under such hypothesis a wide class of PWM power converters can be modeled in the following way:

$\dot{x}(t)=A_{0} x(t)+b_{0}+\sum_{i=1}^{m}\left(A_{i} x(t)+b_{i}\right) n\left(r_{i}(t)-c_{i} x(t)-\delta_{i}(t)\right)$

where $x(0)=x_{0}, A_{i}, b_{i}$ and $c_{i}$ are constant matrices of appropriate dimensions and $m$ is the number of modes 
of the converter. The step nonlinearity $n: \mathbb{R} \rightarrow \mathbb{R}$ is given by

$$
n(z)= \begin{cases}1, & z>0 \\ 0, & z<0 .\end{cases}
$$

The external reference signal $r_{i}(t)$ is assumed to be Lipschitz continuous and the $\delta(t)$ signal is a high frequency signal of period $p$.

It is now possible to derive the averaged system for the feedback PWM system (4) (see Iannelli et al. (2004) for the details).

Proposition 3.1. The averaged system of the pulse width modulated feedback system (4) is given by

$\dot{w}(t)=A_{0} w(t)+b_{0}+\sum_{i=1}^{m}\left(A_{i} w(t)+b_{i}\right) N_{i}\left(r_{i}(t)-c_{i} w(t)\right)$,

where $w(0)=w_{0}$ and $N_{i}(z)=F_{\delta_{i}}(z)$.

PROOF. Follows simply from (2) and

$$
N(z)=\int_{\mathbb{R}} n(z-a) \mathrm{d} F_{\delta}(a)=\int_{-\infty}^{+z} \mathrm{~d} F_{\delta}(a)=F_{\delta}(z) .
$$

Note that, since $n(z)$ is discontinuous in 0 , the averaged nonlinearity $N(z)$ is well defined except at possible discontinuity points of $F_{\delta}$. It means that the right-hand side of (6) is not necessarily well-defined everywhere. The averaged system can have a welldefined generalized solution even if the right-hand side is almost everywhere well-defined. This is the case in the sequel of the paper.

\subsection{Dither effects interpreted through averaged models}

An interesting class of PWM power converters which can be modeled through (4) is represented by DC/DC converters. For instance the voltage-mode controlled buck converter reported in Fig. 1 can be modeled by considering $m=1, A_{1}=0, b_{0}=0, r_{1}=k_{p} V_{\text {ref }}$ and

$A_{0}=\left[\begin{array}{cc}-\frac{R_{1}}{L} & -\frac{1}{L} \\ \frac{1}{C} & -\frac{1}{R_{2} C}\end{array}\right], b_{1}=\left[\begin{array}{ll}\frac{E}{L} & 0\end{array}\right]^{T}, c_{1}=\left[\begin{array}{ll}0 & k_{p}\end{array}\right]$

where $V_{\text {ref }}$ is the output reference voltage, $k_{p}$ the gain of the proportional controller and $\delta(t)$ may be the sawtooth signal reported in Fig. 2. It is simple to show that the DC/DC buck converter can be represented by using the block scheme reported in Fig. 3 with $\alpha=0$ and $G(s)=c_{1}\left(s I-A_{0}\right)^{-1} b_{1}$. The corresponding block scheme of the averaged model is reported in Fig. 4. Let us now consider a DC/DC buck converter with the following parameters: $R_{1}=0.1 \Omega, L=1 \mathrm{mH}$, $C=220 \mu F, R_{2}=8.9 \Omega E=6 V, p=100 \mu s, k_{p}=1.1$ and the reference voltage equal to $6 \mathrm{~V}$ and after $0.03 \mathrm{~s}$ to $8 \mathrm{~V}$. By using as dither signals a sawtooth the results reported in Fig. 5 are obtained. By increasing

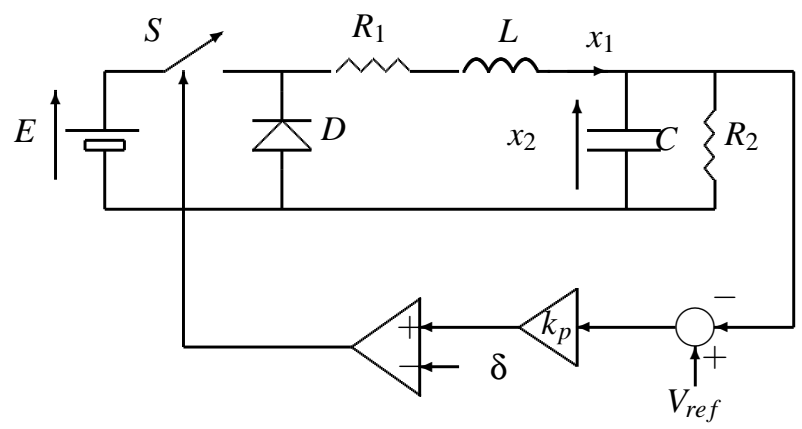

Fig. 1. DC/DC buck converter.
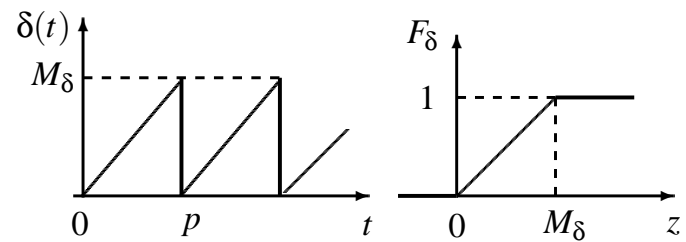

Fig. 2. Sawtooth dither with its amplitude distribution function.

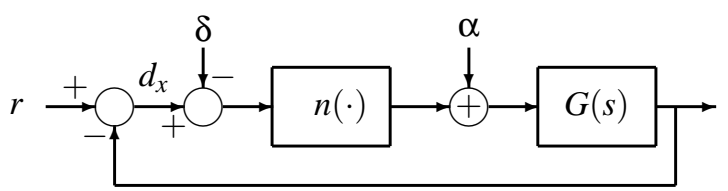

Fig. 3. Equivalent block scheme of a class of power electronic systems; for power converters $d_{x}$ represents the so-called duty cycle.

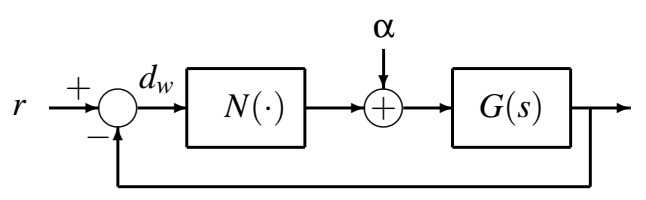

Fig. 4. Block scheme of the averaged model corresponding to Fig. 3.

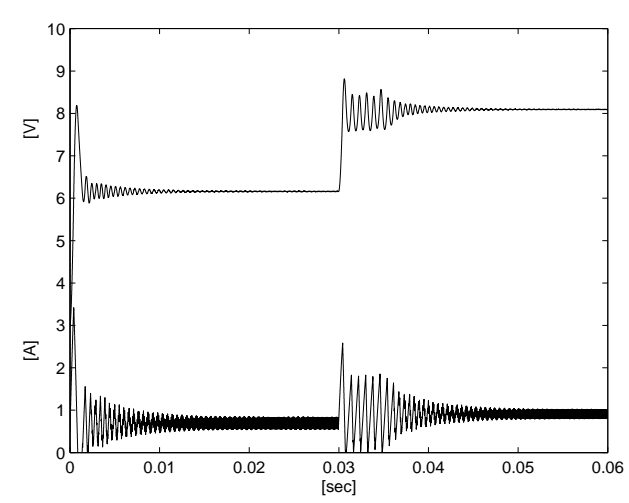

Fig. 5. Capacitor voltage and inductor current of the voltage mode controlled DC/DC buck converter by using a sawtooth dither signal with $M_{\delta}=0.4$. 


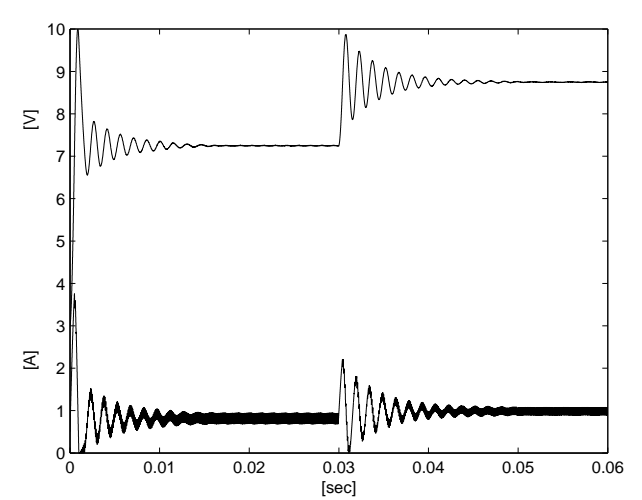

Fig. 6. State variables for the buck converter by using a sawtooth dither signal with $M_{\delta}=4$.

the amplitude of the sawtooth dither signal, the average analysis predicts that the average gain will decrease. Therefore one might expect a degradation of the closed loop performance. This is confirmed by the simulation results reported in Fig. 6. By simulating the power converter and the corresponding averaged model in the presence of a sinusoidal dither, similar results are obtained. Thus, the average analysis seems to work properly also if the averaged nonlinearity is non Lipschitz continuous, so as for the case of the sinusoidal dither to which corresponds an amplitude distribution function with unbounded derivative at $\pm M_{\delta}$. This motivates a further investigation in the need for the assumptions made on the dither signal for the proof of Theorem 2.1.

\section{SUBTLETIES IN AVERAGING RESULTS DUE TO INAPPROPRIATE CHOICE OF DITHER SHAPE}

In this section we will show that when conditions on the amplitude distribution function are not satisfied, it is possible to find systems for which conclusions of Theorem 2.1 do not hold. Our first result shows that we cannot ensure the existence of a unique solution to the averaged system unless we impose the boundedness of the derivative of $F_{\delta}$.

Proposition 4.1. Suppose the amplitude distribution function $F_{\delta}$ is absolutely continuous but its derivative is not bounded. Then there exists a dithered system (1) for which the corresponding averaged system (2) does not have a unique solution.

PROOF. Consider the dithered nonsmooth feedback system (4) with $m=1, A_{0}=A_{1}=0, b_{0}=0, b_{1}=4$, $c_{1}=-1, x(0)=-1, r \equiv 0$ and $\delta$ is the following $p$-periodic quadratic dither signal

$\delta(t)=\left\{\begin{array}{l}-4 M_{\delta}\left(\frac{t}{p}\right)^{2}+M_{\delta}, \quad t \bmod p \in\left(0, \frac{p}{2}\right] \\ +4 M_{\delta}\left(\frac{t}{p}\right)^{2}-8 M_{\delta} \frac{t}{p}+3 M_{\delta}, \quad t \bmod p \in\left[\frac{p}{2}, p\right) .\end{array}\right.$
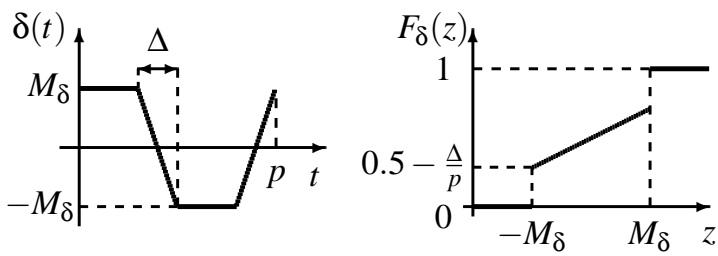

Fig. 7. Trapezoidal dither and the corresponding amplitude distribution function.

The corresponding amplitude distribution function is

$$
F_{\delta}(z)= \begin{cases}0 & z \leq-M_{\delta} \\ \frac{1}{2} \sqrt{1+\frac{z}{M_{\delta}}} & -M_{\delta} \leq z \leq 0 \\ 1-\frac{1}{2} \sqrt{1-\frac{z}{M_{\delta}}} & 0 \leq z \leq M_{\delta} \\ 1 & z \geq M_{\delta} .\end{cases}
$$

Note that the amplitude distribution function is absolutely continuous but its derivative is not bounded. The averaged system is then (6) where $N(z)$ can be obtained from (7) and Proposition 3.1. Note that this averaged system is not globally Lipschitz because $F_{\delta}$ is not globally Lipschitz. It is easy to show that when $M_{\delta}=1$ the averaged system has a nonunique solution. In fact, there are infinitely many solutions parameterized by $\tau \in[0, \infty)$ and given by

$$
w(t)= \begin{cases}-1 & 0 \leq t \leq \tau \\ (t-\tau)^{2}-1 & t \geq \tau .\end{cases}
$$

The next result states that if the amplitude distribution function is not absolutely continuous then the conclusions of Theorem 2.1 cannot be guaranteed.

Proposition 4.2. Suppose the amplitude distribution function $F_{\delta}$ is discontinuous. Then there exists a dithered system (1) for which the uniform bound in (3) of Theorem 2.1 does not hold.

PROOF. Let us consider the nonsmooth feedback system (4) with $m=1, A_{1}$ is a null matrix of adequate dimensions,

$A_{0}=\left[\begin{array}{cc}-1 & -1 \\ 0 & -2\end{array}\right], b_{0}=\left[\begin{array}{c}0 \\ -1\end{array}\right], b_{1}=\left[\begin{array}{l}0 \\ 2\end{array}\right], c_{1}=\left[\begin{array}{ll}1 & 0\end{array}\right]$.

The external constant reference is $r_{1}(t)=R=0.5$ and $\delta$ is a square wave dither of amplitude $M_{\delta}=0.5$. It is easy to show that the averaged system is (6) where $N$ is the averaged nonlinearity corresponding to the amplitude distribution function in Fig. 7 with $\Delta=0$ and $M_{\delta}=0.5$. Let us partition the state space of the dithered and the averaged systems into the following three regions, see Fig. 8:

- Region $\Omega_{1}=\left\{x: x_{1}<0\right\}$. In this region $n(R-$ $\left.c_{1} x-\delta\right)=1$. The dithered system coincides with the averaged system and they have dynamics $\dot{x}=A_{0} x-b_{0}$. The equilibrium point is $P_{1}=$ $+A_{0}^{-1} b_{0}=(-0.5,0.5)^{T}$. 
- Region $\Omega_{2}=\left\{x: x_{1}>1\right\}$. In this region $n(R-$ $\left.c_{1} x-\delta\right)=0$. The dithered system coincides with the averaged system and they have dynamics $\dot{x}=A_{0} x+b_{0}$. The equilibrium point is $P_{2}=$ $-A_{0}^{-1} b_{0}=(0.5,-0.5)^{T}$.

- Region $\Omega_{0}=\left\{x: 0<x_{1}<1\right\}$ with subsets $\Omega_{0}^{+}=$ $\left\{x: 0<x_{1}<1, x_{2}>0.5\right\}$ and $\Omega_{0}^{-}=\left\{x: 0<x_{1}<\right.$ $\left.1, x_{2}<-0.5\right\}$. In $\Omega_{0}$ the state does not affect the output of the step nonlinearity. The dithered system can be represented by the linear system

$$
\dot{\zeta}(t)=A_{0} \zeta(t)-b_{0} u(t)
$$

with $u$ a periodic signal that switches between -1 (when $R-\delta(t)=0$ ) and 1 (when $R-\delta(t)=$ $1)$. The averaged system has an input equal to zero in this region, i.e., $\dot{w}(t)=A_{0} w(t)$.

Consider $x(0)=w(0)$, with $0<x_{1}(0)<1,0<x_{2}(0)<$ 0.5 and $x_{1}(0)>x_{2}(0)$. It is easy to show that the average trajectory will tend to the origin without leaving the set indicated for the possible initial conditions. The dithered trajectory will oscillate about the averaged solution. By considering the vector fields indicated in Fig. 8, it follows that the dithered trajectory cannot leave the set $\Omega_{0}-\left\{\Omega_{0}^{+} \cup \Omega_{0}^{-}\right\}$but by crossing the segment $\left\{x: x_{1}=0,0 \leq x_{2} \leq 0.5\right\}$. This can, for example, be seen by inspecting the phase-plane in Fig. 8. Moreover in $\Omega_{0}$ the solution of the dithered system can be represented as

$$
x(t)=e^{A_{0} t}\left(x(0)-\zeta_{0}\right)+\zeta_{s s}(t),
$$

where $\zeta_{s s}$ is the steady-state $p$-periodic solution of (8) and

$$
\zeta_{0}=\left(I-e^{A_{0} p}\right)^{-1} \int_{0}^{p} e^{A_{0}(p-s)} b u(s) \mathrm{d} s .
$$

Since $A_{0}$ is Hurwitz, $x(t)$ will thus converge to $\zeta_{s s}(t)$, which is a counter clockwise oscillation around the origin. It is always possible to choose a small enough dither period $p$ such that $\zeta_{s s}(t)$ never intersects $\Omega_{2}$, since $\zeta_{s s} \rightarrow 0$ when $p \rightarrow 0$. It is then clear that $x(t)$ eventually will cross the $x_{2}$ axis for some $0 \leq x_{2} \leq 0.5$. ¿From Fig. 8, it is easy to see that the second orthant is an invariant set under the dynamics of the dithered and averaged systems. Moreover, since the system matrix $A_{0}$ is Hurwitz, the dithered solution $x(t)$ will tend toward the equilibrium point $P_{1}$.

We have thus shown that the dithered and the averaged systems behave qualitatively very different since they converge to two different points, $P_{1}$ and the origin, respectively. This is a contradiction to the condition (3) of Theorem 2.1. Indeed, if the compact set $\mathcal{K}$ includes the origin, we would need to make $p$ smaller and smaller the closer $x_{0}$ is to the origin (on the trajectory indicated in Fig. 8) in order to get the inequality satisfied, because it always exists a $p$ such that (3) does not hold. Hence, there is no uniform bound on $p$ that holds for all $x_{0} \in \mathcal{K}$.

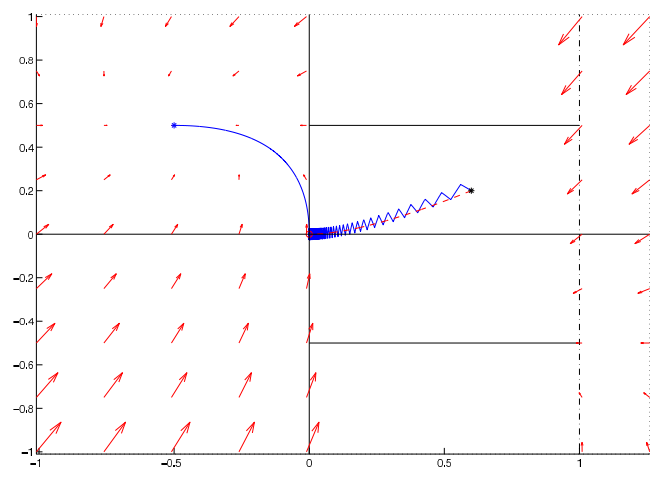

Fig. 8. Phase plane trajectories and vector fields for the dithered system (4) and the averaged system (6) with $p=0.1$ and initial conditions $x_{0}=w_{0}=$ $\left[\begin{array}{ll}0.6 & 0.2\end{array}\right]^{T}$. The trajectory of the dithered system converges to $P_{1}$ while the trajectory of the averaged system converges to the origin.

\section{EXPERIMENTAL RESULTS}

In this section we illustrate how the complex behaviors discussed in previous section may also appear in practical power electronics applications. The experimental setup consists of a power electronic drive with a position controlled DC motor and a full bridge DC/DC power converter. The control objective is to put the motor shaft at a desired angular position $\theta_{\text {ref. }}$. The angular position of the shaft $\theta$ is measured by using a rotational potentiometer whose gain is $k_{\text {pot }}$. The motor supply voltage $\pm V_{a}$ is obtained through a full bridge DC/DC converter. By introducing the state vector $x=\left(\theta, \omega, i_{a}\right)^{T}$, the dynamic model of the whole power electronic system can be represented as the dithered nonsmooth feedback system (4) with $m=1$, $A_{1}$ is a null matrix of adequate dimensions,

$$
\begin{gathered}
A_{0}=\left[\begin{array}{ccc}
0 & 1 & 0 \\
0 & -\frac{\beta}{J} & \frac{k_{t}}{J} \\
0 & -\frac{k_{e}}{L_{a}} & -\frac{R_{a}}{L_{a}}
\end{array}\right], b_{0}=\left[\begin{array}{c}
0 \\
0 \\
-\frac{V_{a}}{L_{a}}
\end{array}\right], \\
b_{1}=-2 b_{0}, c_{1}=\left[\begin{array}{lll}
k_{\mathrm{pot}} & 0 & 0
\end{array}\right] .
\end{gathered}
$$

It is simple to show that such system can be represented through the block diagram reported in Fig. 3 with $\alpha=-0.5$ and $G(s)=c_{1}\left(s I-A_{0}\right)^{-1} b_{1}$. The external constant reference is $r(t)=R=V_{\text {ref. The DC mo- }}$ tor has the following parameters: $R_{a}=2.510 \Omega, L_{a}=$ $0.530 \mathrm{mH}, k_{t}=k_{e}=5.700 \mathrm{mV} /\left(\mathrm{rad} \cdot \mathrm{s}^{-1}\right), \beta=0.411 \mathrm{mN}$. $\mathrm{cm} /\left(\mathrm{rad} \cdot \mathrm{s}^{-1}\right), J=31.400 \mathrm{~g} \cdot \mathrm{cm}^{2}, k_{\mathrm{pot}}=3 /(2 \pi) \mathrm{V} / \mathrm{rad}$, $V_{a}=2.500 \mathrm{~V}$. Two dither shapes are considered: a sawtooth signal and a trapezoidal signal. The dither amplitude is in all cases equal to $M_{\delta}=0.070$. It can be shown (e.g., using the Popov criterion) that the averaged systems corresponding to the sawtooth and trapezoidal dither cases are both asymptotically stable. For sawtooth dither, the approximation error between the dithered system and the averaged system tends to zero as the dither frequency goes to infinity, in accordance with Theorem 2.1. Hence, since the averaged system is asymptotically stable, the system output goes to zero 

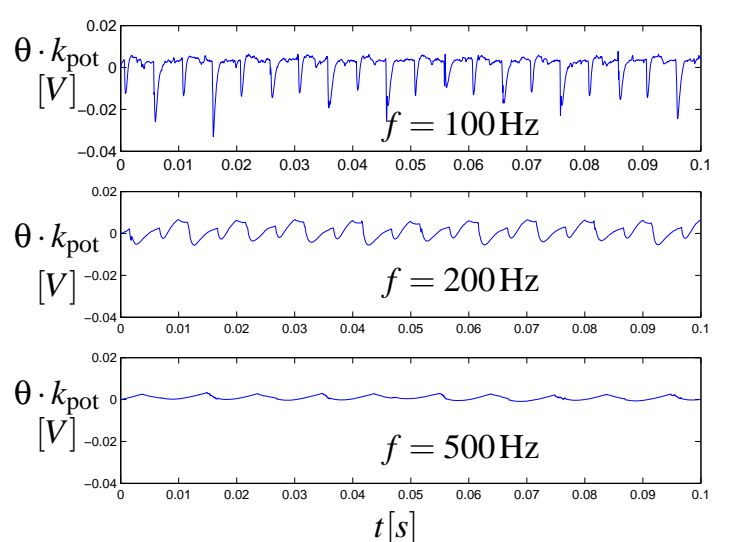

Fig. 9. Angular position for sawtooth dither of three frequencies.

as we increase the dither frequency. For trapezoidal dither, the assumptions of the theorem are not fulfilled, since trapezoidal dither has a discontinuous amplitude distribution function.

The following experiments support these theoretical conclusions. The system is stabilized with sawtooth dither, but not with trapezoidal dither. Experiments were carried out using sawtooth dither of frequencies 100, 200, and $500 \mathrm{~Hz}$. Fig. 9 reports the angular position of the motor shaft under steady-state conditions. Note that by increasing the dither frequency the behavior of the dithered system converges to the behavior of the (stable) averaged system (i.e., the system output goes to zero). The ratio between consecutive averages of the peak-to-peak values of the output signal is equal to 3.33 and 2.84 for $100-200 \mathrm{~Hz}$ and $200-500 \mathrm{~Hz}$, respectively. These ratios somehow indicates the convergence rate. The averaging effect of the dither thus works properly in this case. Fig. 10 shows experiments with trapezoidal dither. In this case, the system output shows a slow oscillation with a substantial amplitude for all three dither frequencies. (Note that the axes are not the same as in Fig. 9.) The frequency of the oscillation is low compared to the dither frequency, and it seems to be relatively independent of the dither frequency. In particular, note that by increasing the dither frequency, the system output does not converge to zero, as was the case with sawtooth dither. Instead the ratio between consecutive averages of the peak-topeak values of the output signal is equal to 1.97 and 0.86 for $100-200 \mathrm{~Hz}$ and $200-500 \mathrm{~Hz}$, respectively, so going from 200 to $500 \mathrm{~Hz}$, the amplitude of the oscillation is even increasing.

\section{CONCLUSIONS}

In this paper we have shown that an important class of power electronics systems (namely pulse width modulated systems), usually modeled as hybrid dynamical systems with external periodic forcing, can be recasted in the framework of dithered nonsmooth systems. By exploiting recent theory presented by authors on the averaging of dithered nonsmooth systems, we have an-
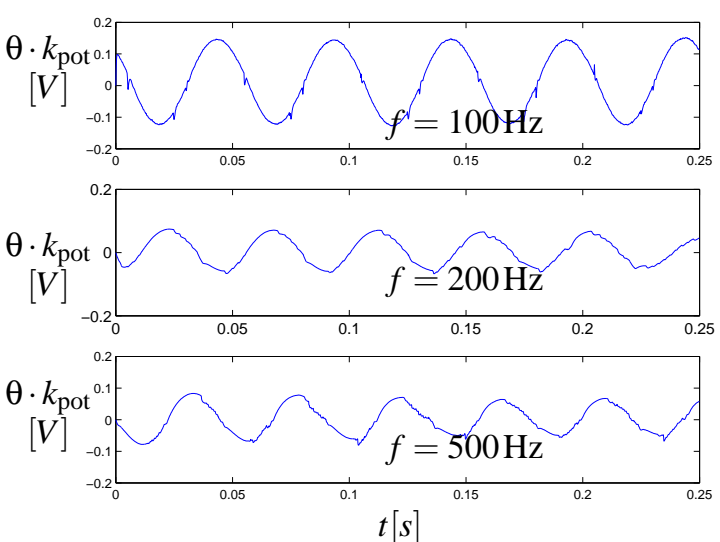

Fig. 10. Angular position for trapezoidal dither of three frequencies. Note the different scale compared to Fig. 9.

alyzed how the shape of the external forcing might affect the averaging result and the behavior of the overall system. Simulations and experiments supported the theoretical discussion and proved the importance of averaging theory and its subtleties in the case of its application to hybrid dynamics.

\section{REFERENCES}

S. Banerjee and G.C. Verghese, editors. Nonlinear Phenomena in Power Electronics: Bifurcations, Chaos, Control, and Applications. Wiley-IEEE Press, New York, 2001.

M. di Bernardo and C.K. Tse. Complex behavior in switching power converters. Proceedings of the IEEE, 90(5):768-781, 2002.

A.Kh. Gelig and A. Churilov. Stability and Oscillations of Nonlinear Pulse Modulated Systems. Birkhäuser, Berlin, 1998.

L. Iannelli, K. H. Johansson, U. Jönsson, and F. Vasca. Averaging of nonsmooth systems through dithered switchings. Automatica, 42(4):669-676, 2006.

L. Iannelli, K.H. Johansson, U. Jönsson, and F. Vasca. Dither for smoothing relay feedback systems: an averaging approach. IEEE Transactions on Circuits and Systems, Part I, 50(8):1025-1035, 2003.

L. Iannelli, K.H. Johansson, U. Jönsson, and F. Vasca. On the averaging of a class of hybrid systems. In Proc. of IEEE Conference on Decision and Control, volume 2, pages 1400-1405, Bahamas, 2004.

A. Teel, L. Moreau, and D. Nesic. Input-to-state set stability of pulse width modulated systems with disturbances. Systems and Control Letters, 51, 2004.

C.K. Tse, Y.M. Lai, and H.H.C. Iu. Hopf bifurcation and chaos in a free-running current-controlled Ćuk switching regulator. IEEE Transactions on Circuits and Systems, Part I, 47(4):448-457, 2000.

G. Zames and N. A. Shneydor. Dither in non-linear systems. IEEE Transactions on Automatic Control, 21(5):660-667, 1976. 